\title{
L'engraissement des zébus dans la région de Tananarive selon la technique du "bœuf de fosse »
}

\author{
par H. SERRES
}

\begin{abstract}
RÉSUMÉ
Aux environs de Tananarive, on engraisse des zébus maigres en les plaçant dans des « fosses » creusées à flanc de penie autour des villages. De décembre à mars les animaux sont nourrıs d'herbe, ensuite de mars à juillet de repousses de riz, fevilles de patates et manioc. Les animaux deviennent très gras avec un rendement en carcasses atteıgnanf 65 p. 100. Cette activité associée à la riziculture devrait être multipliée, mais il semble que l'exıguïté des propriétés soit, dans la région considérée, un facieur limitant.
\end{abstract}

Les abattorrs de Tananarive utilisent des bovins qui proviennent pour la plupart des régions de l'Ouest de l'île. Ces animaux arrivent à pied à la capitale dans un état médiocre d'embonpoint. Comme il existe une clientèle pour de la viande grasse et même très grasse, une activité s'est développée de façon traditıonnelle dans les villages qui entourent la ville de Tananarive, à plusieurs dizaines de kilomètres à la ronde. Les paysans engraissent des baufs achetés maigres selon une technique qui est dite du «bouf de fosse ». La plupart de ceux qui ont écrit sur l'élevage bovin à Madagascar en ont fait mention, mais il n'existe à notre connaissance aucun document précis sur ce mode d'élevage. Devant l'importance économıque de la question, nous avons essayé de donner quelques chiffres et de lever un tant soit peu le mystère qui entoure le bcuf de fosse. A vrai dire ce ne fut pas chose facile car les paysans se livrent peu. Pendant une année entière nous avons localısé notre enquête sur le village d'Ampahimanga, situé à l'Est de Tananarive et voisin du marché d'Ambatomanga où se vendent chaque année de beaux spécimens.
Progressivement nous avons pu gagner suffisamment la confiance des éleveurs pour obtenir des précisions que nous allons décrire ci-après et nous verrons que la pratique procède d'une expérience solidement fondée.

\section{L'ACHAT DES ANIMAUX}

Les animaux sont acheiés de façon générale à la fin de la saison sèche, en octobre. Le choix n'en est nullement indifférent. Des marchands de bétail spécialisés vont acheter des bêtes au grand marché d'Ambalavao situé à la lımite Sud des hauts plateaux et les amènent dans la région de Tananarive où les paysans les rachètent. Les anımaux les plus estimés proviennent des régions Sud de Madagascar et notamment de l'Androy.

On recherche des anımaux âgés, ayant terminé leur croissance ef parfois même très âgés : entre 8 et 12 ans en règle générale. Les grandes carcasses sont les plus prisées. De même, on recherche les animaux ayant au plus haut degré des caractères de finesse : tête légère avec un mufle petit et des lèvres fines formant un museau 


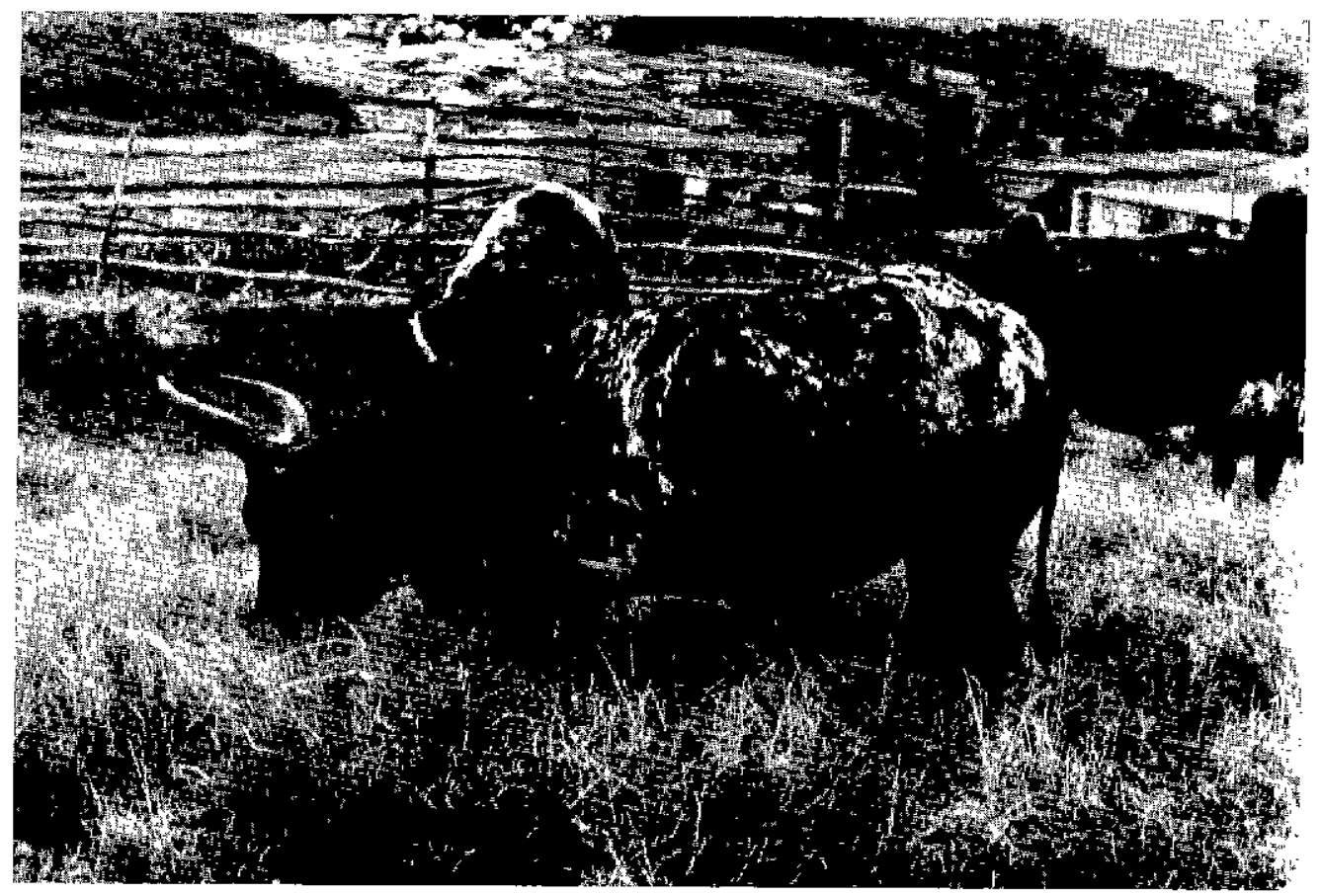

Photo no 1. - Bøuf de fosse de petit format - Taille au garrot $123 \mathrm{~cm}$. Poids $430 \mathrm{~kg}$.

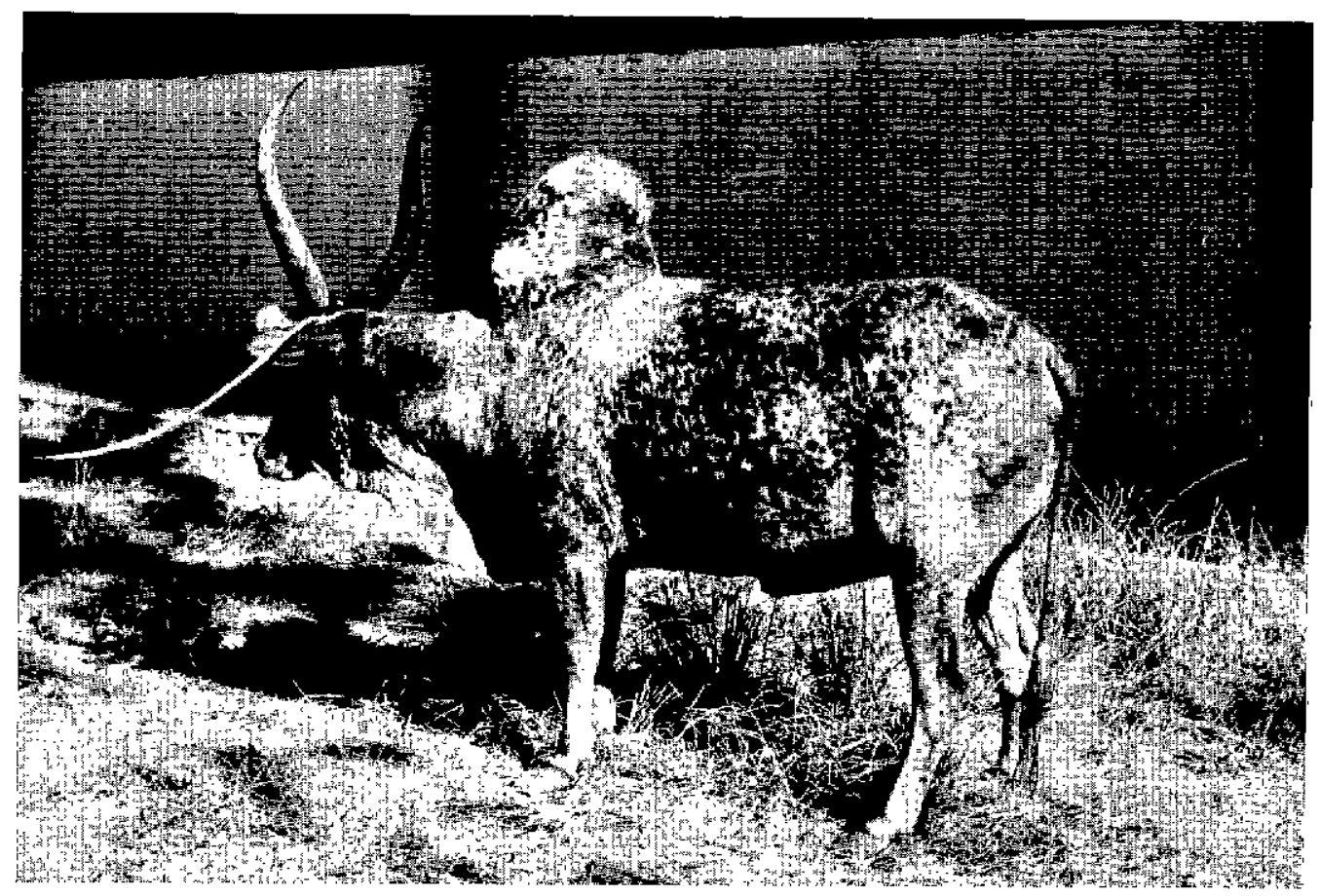

Phota no 2. - Bcuf de fosse de format moyen - Talle au garrot $127 \mathrm{~cm}$. Poids $450 \mathrm{~kg} \mathrm{-}$ Noter la finesse générale du squelette. 


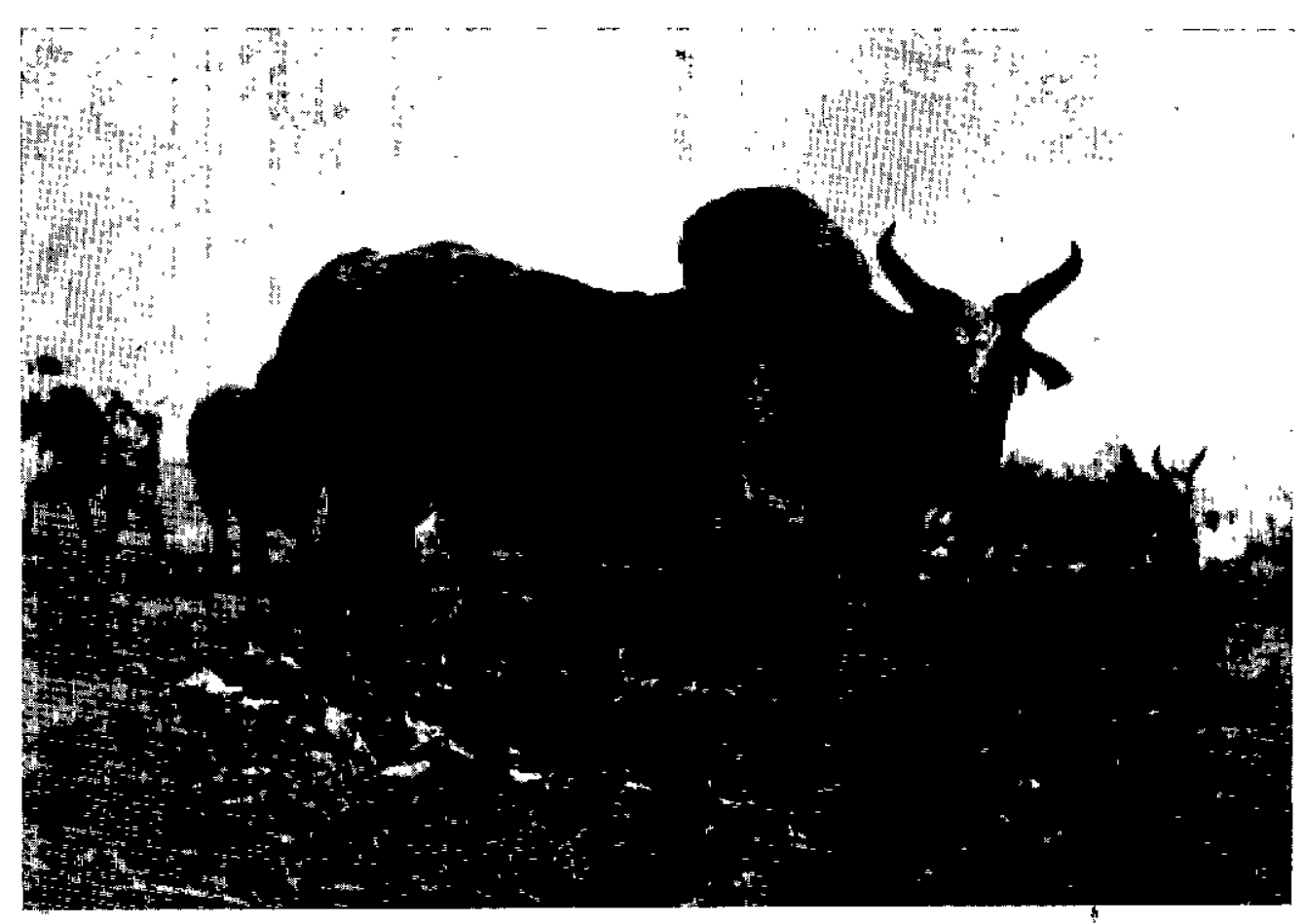

Photo no 3. - Bouf de fosse de grand format - Taille ou garrot $133 \mathrm{~cm}$ Animal très long et profond. Poids $500 \mathrm{~kg}$.

pointu; cornes longues et effilées, membres fins, queve longue et fine.

L'état de bonne santé des animaux est jugé en les faisant courir le long d'une côte. Au sommet, ceux qui toussent ou qui soufflent anormalement sont éliminés. Nous avons pu avoir la certitude que ce procédé était efficace.

Au sujet du choix du bétall on peut déjà faire quelques commentaires : les animaux âgés ont un squelette déjà élaboré et leurs besoins phosphocalçiques sont limités, ce qui favorisera l'opération dans un milleu très pauvre en calcium.

On \$ait, zootechnıquement parlant, que les animaúx fins ont corrélativement plus d'aptitude à faire de la graisse que les autres : le choix traditionnel se trouve donc en accord avec nos connaissances techniques pour le but recherché.

L'origine des anımaux est aussi à commenter.

L'extrême Sud de Madagascar est la région oủ la pluviométrie est la moins abondante et où la saison sèche est la plus longue. Les anımaux s'y sont donc adaptés par sélection naturelle à profiter au maximum de la courte saison favorable pour mettre rapıdement en réserve le maximum d'énergie pour passer la longue période défavorable. Cette aptitude est mise à profit pour obtenir un bon engraissement.

\section{TECHNIQUE DE L'ENGRAISSEMENT DU BCEUF DE FOSSE}

Période préliminarre : les boufs récemment achetés sont d'abord utilisés au î́nois de novembre pour le piétinement des rizıères qui remplace le hersage. Les anımaux effectueght ce travail sans autre alimentation que le pâturage naturel. Ils maigrissent et se fatıguent. Lés paysans y voient un double intérêt : d'abord l'utilisation pour un travail qui se falt seulement une fois I'an pendant une quinzaine de jours, ensuite la fatigue des animaux qui rend leur adaptation plus facile à la claustration ef va accroître leur appétit dons les jours suivants?

$$
\text { Période d'engraissếment }
$$

Le bouf est mis dans uñe fosse où il séjournera pendani toute la duréę de son engrassement. 

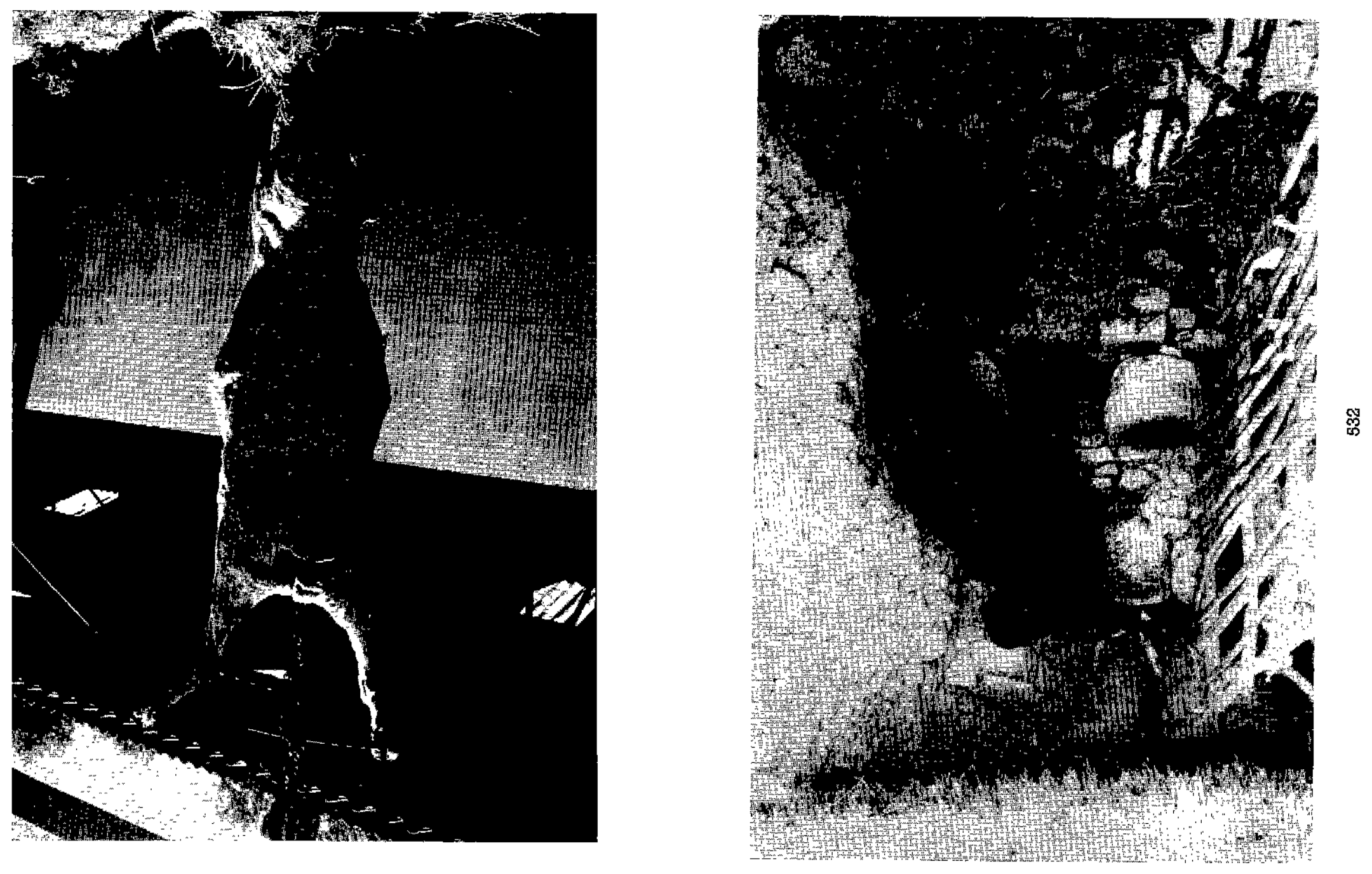


\section{A) Description d'une fosse.}

Elle est creusée au flanc d'une pente, ce qui, du côté inférieur, permet un accès plus ou moins facile pour l'entrée et la sortıe du bœuf. Les fosses, distribuées autour du village, sont généralement situées sur une pente orientée à l'Ouest afin que les animaux soient à l'abri de l'alizé qui souffle du Sud-Est et bien ensoleillés depuis le milieu de la matinée jusqu'au sorr, alors que le soleil est chaud. A cause de cet ensoleillement, jugé nécessaire, les fosses ne sont jamals couvertes malgré les pluies diluviennes.

Leurs dimensions sont variables, mais souvent le bouf dispose d'une vingtaıne de mètres carrés selon un rectangle plus ou moins régulier. II arrive que des fosses plus grandes soient divisées par une barrière et reçolvent deux bœufs. Chaque bouf est toujours isolé pour éviter que l'un n'empêche l'autre de manger.

Le sol de la fosse a une pente assez accusée de manière à ce que l'eau de pluie puisse s'écouler après avoir lavé la litière du bœuf. Elle est d'ailleurs utılısée en dessous de la fosse pour la fertilisation du chomp de patates douces que nous retrouverons plus loin.

Les fosses sont plus ou moins complètement équipées. Les plus raffinées disposent d'un «bloc d'alimentation » très étudié. Il est essentiellement constitué par une mangeorre en pierre, toujours située en hauteur et à laquelle l'anımal ne peut accéder qu'en plaçant les antérieurs sur une marche. Un cornadis fait de deux pierres plates placées verticalement isole la tête du bœeuf lorsqu'il mange. Au-dessus de la mangeorre se trouve une plate-forme accessible au paysan par le bord supérieur de la fosse, où sont placés les aliments que l'on va distribuer par fractions au bœuf.

Auprès de la marche donnant accès à la mangeoire est placé un abreuvorr monolithique rond, dans lequel l'eau est renouvelée depuis le haut.

L'ensemble «bloc d'alimentation » est couvert en chaume de manière à ce que les aliments ne soient pas endommagés par les intempéries.

Dans toutes les fosses, la mangeorre est en position élevée pour oblıger le bcuf à se nourrir tête haute. Les raisons données par les paysons sonf diverses et peu convaincantes : entre autres faciliter la déglutition. II faut remarquer que le système, surtout lorsqu'il est pourvu d'un cornadis, est parfaitement adapté à la lutte contre le gaspillage. Le bœuf ne peut faire de grands mouvements de tête qui projettent toujours des aliments hors de la mangearre. On peut penser ensuite que le fait de répéter l'ascension vers la mangeoire favorise une musculation de l'arrièretraın. On remarque, en effet, que les bœufs de fosse ont tous une fesse très rebonidie que le zébu d'herbe ne présente jamais.

A la partie postérieure de la fosse se trouve la porte qui permet à l'anımal de sortir en empruntant une sorte de couloir ou d'escalier. Cette issue est toujours soigneusement barricadée ovec des branchages entremêlés. Une fois en fosse, le bouf n'en sort plus que pour la vente: il est donc inutile que la porte soit mobıle, et le propriétaire souhaite même le contraire pour éviter un vol.

\section{B) Alimentation du bcuf de fosse.}

Le bœuf qui vient d'être enfermé n'est pas habitué à recevoir une nourriture intensive non pâturée. II y a une période d'adaptation qui dure environ un mois. On donne d'abord de la paille de riz au bœuf, puis de l'herbe coupée qu'il consomme de plus en plus volontiers. Lorsqu'il y est habitué on ne lui donne plus que de l'herbe. Cette herbe de début de saison des pluies est coupée sur les talus, les bas de pentes, les diguettes de rizières. Elle est mise en bottes et transportée au village, sur la tête, en ballots d'environ $20 \mathrm{~kg}$ chacun.

Les plantes qui composent cette herbe fine sont dominées par :

\section{Cynodon dactylon \\ Setaria pallidefusca \\ Digitaria horizontalis}

S'y mêlent un peu de petit riz poussant sur les diguettes et quelques autres graminées sans importance pondérale.

A cette herbe très humıde ( 85 p. 100 d'eau), on ne peut attribuer plus de 0,12 Unité fourragère au $\mathrm{kg}$. Par contre, sa teneur en matıère azotée est élevée, l'analyse donne 2,4 p. 100 sur produit frass soit 16 p. 100 sur la matière sèche.

On arrive à faire manger au bœuf $60 \mathrm{~kg}$ de cette herbe chaque jour, ce qui donne le bilan suivant : 
7 Unités fourragères

$9 \mathrm{~kg}$ de matière sèche

$1.440 \mathrm{~g}$ de matlère azotée brufe

MAB/U. F. $200 \mathrm{~g}$.

On se trouve donc en présence d'une ration abondante sur le plan énergétique et riche en matières azotées brutes ainsi qu'en carotènes.

Les animaux qui n'arrivent pas à développer leur appétit pour consommer une ration de cet ordre sont éliminés et revendus.

Ce type d'alimentation se poursuit pendant les mois de janvier, février et mars qui sont pluvieux.

Pendant cette période, les animaux prennent des formes, un poil brillant, se musclent, mais on ne distingue pas d'hypertrophie des tissus graisseux.

Le premier tournant se situe en fin mars. Les premiers riz ont été récoltés en février et l'on va disposer des repousses de riz qui vont participer de plus en plus à l'alimentation du bouf. Vers la même époque se prépare la récolte des arachıdes. Quelques jours avant celle-ci, les fanes encore très vertes sont coupées pour être distribuées au bœuf qui reçoit encore de l'herbe fine. Pendant un certain temps on va trouver une ration d'environ $60 \mathrm{~kg}$ qui est composée par tiers d'herbe fine qui n'esł plus aussi riche en matières azotées que précédemment car elle a môr, et où l'on rencontre, en plus des plantes précitées, quelques cypéracées fines et quelques composées; par tiers de fanes d'arachides et par tiers de jeunes repousses de riz qui sont à ce moment-là riches en matières azotées. De sorte que le bilan nutritionnel est peu modifié par rapport au précédent. Mais cela ne dure pas: les fanes d'arachides s'épuisent et les repousses de riz évoluent en perdant de leur valeur azotée.

Au mois d'avril, commence la période d'engraissement avec l'apparition du manioc dans la ration. Les herbes fines sont épulsées. On rencontre des rations du type suivant :

Repousses de $r \mid z \ldots \ldots \ldots \ldots 30 \mathrm{~kg}$

Feuilles ef lianes

de patates ............. $10 \mathrm{~kg}$

Manioc vert ............ $5 \mathrm{~kg}$

Ce qui donne une ration d'environ $7 \mathrm{U}$. F. mais avec un peu moins de matière sèche $(8 \mathrm{~kg}$ environ) et surtout bequcoup moins de matière azotée, le rapport MAB/U. F. passant aux environs de $100 \mathrm{~g}$.

Les feuilles de patates sont coupées dans les champs qui se trouvent en dessous des fosses et qui ont bénéficlé de l'apport des purins. De façon générale, les feuilles et lianes de patates sont distribuées fraîches, mais il arrive parfols que les bœufs les apprécient mieux après un préfanage. De toute manière, la quantité distribuée reste la même, à l'humidité près.

Des feurliles de manioc sont mélangées aux lanes de patates en quantité variable mals en général faible. Ces feuilles sont riches en azote.

Le manioc est arraché chaque jour et distribué frais aux animaux, de préférence le soir, la verdure étant donnée dans la journée en plusieurs fols.

En mai, juin, juillet, se développe l'engralssement proprement dit, caractérisé par l'augmentation des distributions journalières de manioc frais.

Nous avons relevé les distributions journalières suivantes:

Manioc ........................ $10 \mathrm{~kg}$

Repousses de riz........... $15 \mathrm{~kg}$

Feuilles et lianes de patates.... $15 \mathrm{~kg}$

Les repousses de riz sont alors jounissantes ef cellulosiques à cause du froid, leur teneur azotée est diminuée fortement. Les feuilles de patates, par contre, conservent un aspect ef une composition assez constants.

Parfols les paysans arrivent à trouver et distribuent une petite quantité d'herbe grossière ou I'on note Hyparrhenia rufa et Panicum maximum coupés sur les pentes autour des villages.

On note alors des refus dans les aliments distribués au bcuf, et ce sont surtout les repousses de riz qui sont délaissées.

Les rations sont d'environ 8 U. F. par jour avec $9 \mathrm{~kg}$ de matière sèche mais la matière azotée devient de plus en plus basse et se situe en dessous de $50 \mathrm{~g}$ de matières azotées brutes par Unité fourragère.

Au cours de cette période, où l'alimentation est surtout énergétique, les tissus gras de l'animal s'hypertrophient. La bosse prend un volume considérable mais demeure néanmoins dressée. Elle déborde sur les épaules et sur l'encolure. A la marche, elle dodelıne au rythme des pas. Le tronc devient cylindrique ef empâté, la fesse 
prend un profil très convexe et une masse graisseuse s'accumule au périnée sur les anımaux extra gras. Les caractères de finesse de la tête, de l'encolure et des membres n'en deviennent que plus accusés.

En fin juillet, les animaux sont au maximum d'engraissement. A ce moment-là il n'y a plus d'herbe, plus de feuilles de patates, les repousses de riz sont pailleuses et il ne reste que du manioc, insuffisant pour assurer l'équilibre de la ration.

\section{Commentaires sur l'alimentation}

L'alimentation qui est distribuée aux boufs dès qu'ils sont habitués contient une quantité élevée d'énergie : $7 U$. F. Pour un animal de $350 \mathrm{~kg}$, bientôt $400 \mathrm{~kg}$, elle laisse pour l'engraissement un disponible voisin de 4 U. F. par jour qui dolvent pouvorr procurer $700 \mathrm{~g}$ de gain, si compte tenu de l'âge avancé des animaux, on estime à 5,5 U. F. le kg de gain (Souf en fin d'engrasssement où le dépôt de graisse est plus onéreux).

Au cours de l'engralssement terminal, ce niveau énergétıque tend à s'élever ( 8 U. F.) par apport de manioc, ce qui est rationnel.

L'apport de manioc dans les derniers mols abaisse l'encombrement de la ration et les réservoirs digestifs diminuent d'importance. Cela contribue à l'obtention d'excellents rendements en carcasses.

Les rations ont, au cours du début de l'engralssement, une teneur en matière azotée élevée qui permet un accroissement muscularre; cette matière azotée diminue progressivement et cela est également rationnel.

La seule ombre au tableau est l'ınsuffisance en minéraux qui sans doute est l'un des facteurs imposant l'emploi d'anımaux adultes.

\section{PERFORMANCES D'ENGRAISSEMENT}

Nous avons eu davantage de difficultés pour peser des animaux que pour contrôler la nourriture qui leur est distribuée. Les propriétaires refusent d'amener les anımaux ò la bascule.

Nous avons réussi à peser 8 animaux au moment où, après le piétinage des rizières, ils allaınt être mis dans les fosses à leur poids le plus bas. Nous donnerons ci-après leur poids et leur taille au garrot. $\begin{array}{lllllllll}\text { No } & 1 & 2 & 3 & 4 & 5 & 6 & 7 & 8\end{array}$

- - - - - - - - -

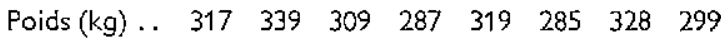

Taille $(\mathrm{cm})$. $\begin{array}{llllllll}127 & 129 & 119 & 123 & 129 & 127 & 132 & 127\end{array}$

De ces quelques chiffres on peut tirer que les animaux les plus grands sont les plus lourds ( $N$ os $2,5,7)$. Si on considère que la taille moyenne au garrot du zébu malgache est de $125 \mathrm{~cm}$, on remarque que six sur huit animaux sont au-dessus. Ces chiffres, insuffisants, matérialisent quand même l'attrait des engraisseurs pour les animaux de grand format quand ils ont les moyens de les acheter.

Nous avons pu examiner les bceufs en fin d'engraissement au marché d'Ambatomanga qui se tient au début de jullet et où 150 bœufs gras étalent présentés. II nous a été impossible de passer à la bascule les anımaux autres que ceux que nous avons achetés. Deux animaux choisis au-dessus de la moyenne pesaient 465 ef $450 \mathrm{~kg}$.

Ces deux anımaux nous ont servi de terme de comparalson pour l'appréciation de l'ensemble du bétail. On doit d'ailleurs distinguer selon deux critères : l'état d'engraissement et le poids.

II semble que le quart environ des animaux puisse être qualifié d'«extra gras»: bosse énorme et débordant en bourrelet à l'avant et sur les côtés, peau du tronc tendue par une gralsse de couverture épaisse qui cache toutes les formes musculares dorsales, accumulation de graisse périnéale.

Plus de la moitié des anımaux sont « très gras » bosse très volumineuse mais peu ou, pas débordante, peau dorsale moins tendue, graisse périnéale peu abondante.

Quelques animaux sont simplement «gras», en très bon état, avec une belle bosse mais sans véritable hypertrophie des tissus graisseux.

Quant au poids, il est fonction de l'état d'engraissement mais plus encore de la taille des individus.

Les animaux «extra gras» se situent entre 430 ef $480 \mathrm{~kg}$. Quelques bœufs exceptionnels afteignent ou dépassent $500 \mathrm{~kg}$. Ils sont rares ef ce sont des animaux remarquables par leur stature avec une taille au garrot approchant $135 \mathrm{~cm}$ et un corps long et profond.

Les animaux «très gras» sont un peu plus légers mais assez peu : de 420 à $470 \mathrm{~kg}$. Enfin, 
les animaux seulement «gras » pèsent de 380 à $430 \mathrm{~kg}$.

II semble que la plupart des bœufs de fosse soient capables de prendre entre 100 et $120 \mathrm{~kg}$ en 200 jours, soit de 500 à $600 \mathrm{~g}$ par jour. Quelques animaux exceptionnels peuvent avolr des performances légèrement supérieures. A I'inverse nous devons signaler un certain déchet : après quelques semaines en fosse, si un animal ne développe pas l'appétit lui permettant de consommer près des $7 \mathrm{U}$. F. requises chaque jour, il est éliminé et revendu. Cela peut se produire pour un animal sur dix, voire plus.

L'indice de consommation moyen en U. F. par $\mathrm{kg}$ de gain se situe entre 13 et 14 .

\section{ÉTUDE DES CARCASSES}

Au marché d'Ambatomanga nous avons acheté deux boufs que nous avons abattus et dont la découpe a été faite avec la collaboration de J. GILIBERT ef TARDIF J.

Le bouf $n^{0} 1$ représentait la catégorie «très gras 》 le bœuf no 2 la catégorie « extra gras» :

Le bouf no 1 avait $129 \mathrm{~cm}$ au garrot;

Le bœuf $n^{\circ} 2$ avait $127 \mathrm{~cm}$ au garrot.

Les résultats de la découpe sont rassemblés au tablecu I.

Les deux animaux étaient très couverts de graisse, de façon un peu plus épaisse pour le

TABLFAU $\mathbb{N}^{\circ}$ I

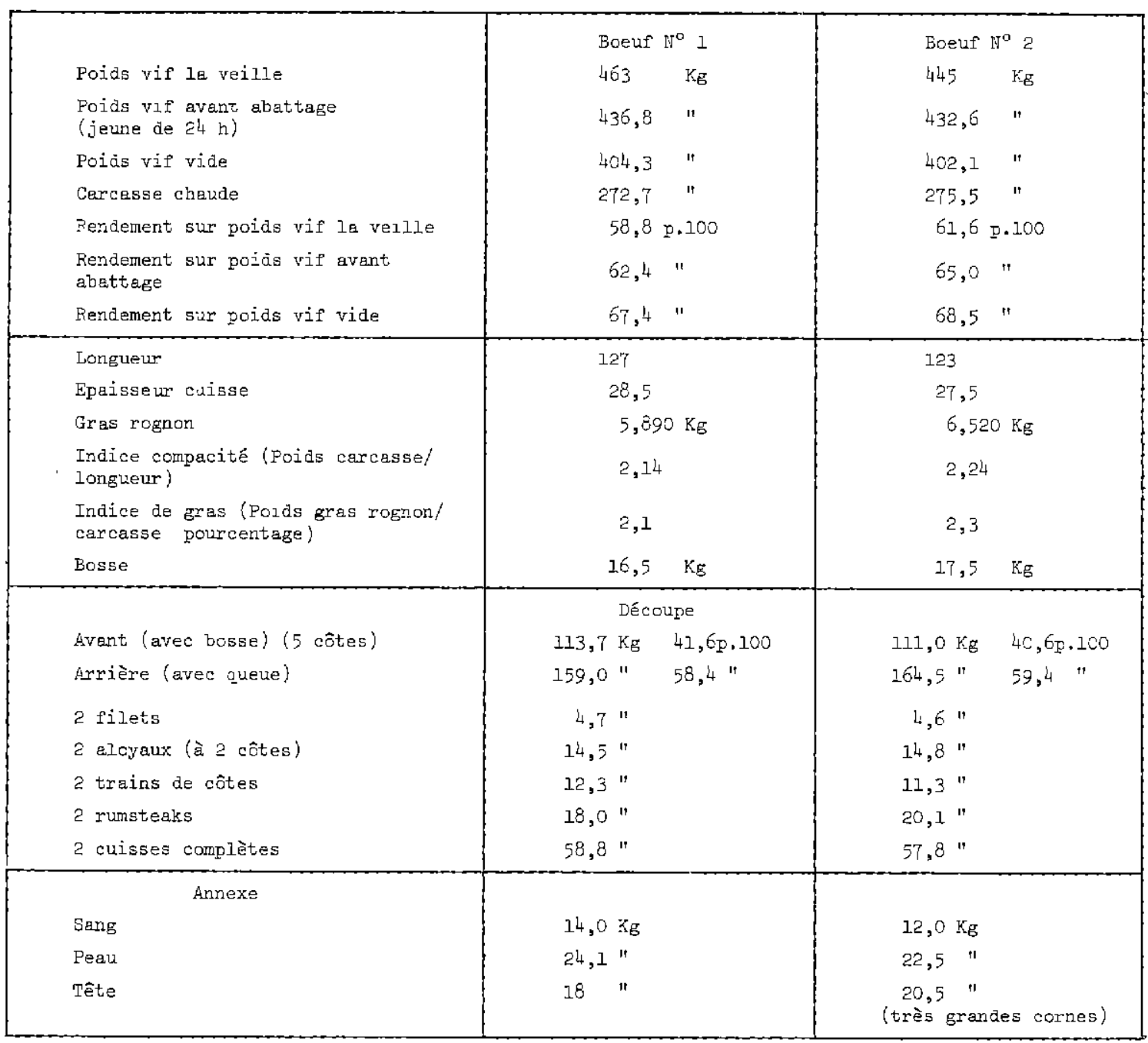




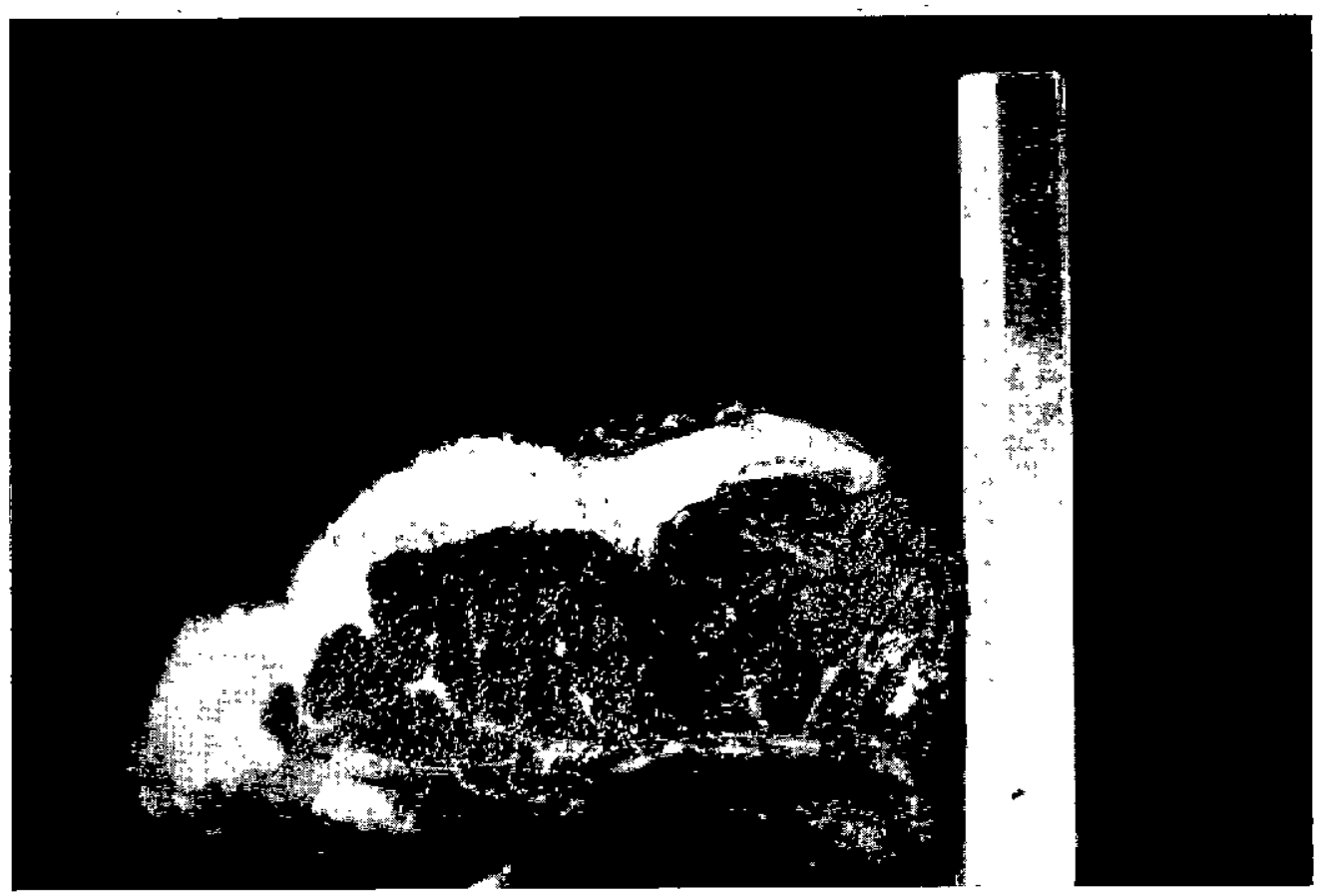

Photo $n^{0}$ 6. - Coupe du long dorsal - Grasse d'infiltration intramusculaire importante et couverture très épaisse.

n०2. Pour le consommateur et le boucher européens elle est en excès. L'infiltration graisseuse intramusculaire est importante.

Si l'on compare les deux carcasses, on remarque que l'extra gras a une bosse et un gras de rognons plus lourds, un rendement en carcasse plus élevé. A souligner qu'il atteint 65 p. 100 sur poids vifavant abattage. La proportion del'arrière par rapport à l'avant est plus favorable.

Nous devons dire ici un mot des prix : le bœuf $n^{\circ} 1$ a été payé $29.000 \mathrm{Fmg}$, et le $n^{\circ} 236.000 \mathrm{Fmg}$ (après marchandage serré).

Ce qui donne sur pied :

$80 \mathrm{Fmg} \mathrm{le} \mathrm{kg} \mathrm{pour} \mathrm{l'extra} \mathrm{gras.}$

$63,5 \mathrm{Fmg}$ le $\mathrm{kg}$ pour le très gras.

Si l'on compare ces prix avec les résultats de l'abattage, on ne peut s'empêcher de remarquer que la différence de l'état de gras est payée bien cher : elle ne peut s'explıquer que par un attrait traditionnel très fort pour la viande grasse au maximum.

\section{APERÇU ÉCONOMIQUE}

Le bœuf coûte à l'achat entre 15.000 et 20.000 Fmg sulvant son format. Quelques animaux très grands ef très prometteurs peuvent coûter jusqu'à 24.000 F mais de façon très exceptionnelle.

On peut tabler sur un prix d'achat moyen de $18.000 \mathrm{Fmg}$, prix élevé pour des animaux maigres et qui ne se justifie que par leur origine lantaine et une succession d'ıntermédıaires.

La moyenne des prix de vente ne dépasse pos beaucoup $30.000 \mathrm{Fmg}$. Le bénéfice réalisé se situe aux environs de $12.000 \mathrm{Fmg}$ avec une variation en plus ou en moins selon que l'animal aura été bon ou médiocre (Nous avons vu le cas d'un animal acheté 20.000 et revendu 40.000 ).

Pour ce gain, il faut considérer que l'entretıen d'un bœuf demande au moins deux heures de travall par jour pour couper l'herbe, les repousses de riz, cultiver le manioc et le récolter, etc. Cela fait qu'un foyer engraisse en général un bceuf. Si le foyer a de grands enfants qui peuvent aider on engraisse alors deux bœufs. A souligner que ce travail est fait par les hommes.

A raison de $500 \mathrm{~h}$ de travail par bouf la rémunération horaire se situe aux environs de $25 \mathrm{Fmg}$, avec toujours une variation en plus ov en , moins.

On peut comparer ce chiffre avec celui du SMIG agricole de la région qui est de $16 \mathrm{Fmg} / \mathrm{h}$. 
Au bénéfice réalisé par la revente dü boeuf engraissé il ne faut pas omettre d'ajouter la valeur du fumier qui s'est accumulé dans la fosse sur une épaisseur pouvant atteindre $80 \mathrm{~cm}$ voire $1 \mathrm{~m}$ à la partie postérieure de la fosse. La quantité de ce fumier peut être de 3 à $5+i$ il sera épandu sur les champs de manioc pour partie, mas également dans la rizière.

Le fumier apporte dans ces derniers cas une recette supplémentaire difficile à chiffrer. On peut néanmoins signaler qu'en banlieve de Tananarive le fumier se vend de $1 \mathrm{Fmg}$ à $1,5 \mathrm{Fmg}$ le $\mathrm{kg}$.

Le purin des fosses engraisse les champs de patates dont les feulles vont au bœuf mais les tubercules au paysan.

Une étude plus serrée des temps de travaux ef de l'importance du fumier permettrait de préciser les termes iéconomiques de l'engralssement des boufs de fosse.

\section{DISCUSSION}

L'engraissement traditionnel des boeufs en fosse procède d'une expérience très affinée qui, par beaucoup de points, est parfaitement logique dans le cadre où elle se déroule. II ne faut pas oublier que l'activité fondamentale du paysan est la riziculture à laquelle sont réservées toutes les terres pouvant être mises en eau.

Les bœufs servent au piétinage de la rizıère et à la fumure. La rizlère rend au bouf les repousses de riz (d'ailleurs très peu denses : $1,5 t$ à l'ha). Le paysan utilise les herbes de colluvions et de diguettes que la nature fait pousser pendant les pluies, puis les fanes d'arachides et les feuilles et lianes de patates cultivées pour la consommation. La seule culture vraiment faite pour le bœuf est le manioc. II n'y a aucun mystère dans l'alımentation des bœufs de fosse : les paysans donnent à l'animal ce dont Ils disposent au fur et à mesure que la saison avance. Cela implique que des variations soient possibles avec les régıons si les disponibles eux-mêmes varient.

L'anomalie réside en ce que cette actıvité est interrompue une partie de l'année, faute d'aliments pour le bétail, alors même que le paysan n'est pas occupé par la riziculture. Des solutions se présentent à l'esprit telles que les fourrages à contre-saison, la création de réserves fourrọgères entre autres, pour permettre au paysan d'engraisser un deuxième bceuf dans l'année.

Avant d'être totalement affirmatıf sur une méthode, il convient d'examiner toutes ses conséquences sur les autres activités d'u paysan et notamment la riziculture, secteur prioritaire dans la tradition.

Il ne faudra pas omettre d'examiner également les surfaces disponibles pourla culture du manioc. A première vue il semble que nombreux sont les propriétarres dans cette régıon qui ne disposeraient pas d'assez de terres aptes à la culture du manıc pour permettre l'engralssement de deux bocufs.

La finition de deux boufs serait facilitée si l'engraissement était poussé moins loin et visait à faire des animaux bien finis mais non hypergras.

Malheureusement, les prix du marché très élevés, comme nous l'avons vu, pour les animaux très gras, sont un facteur défavorable pour cette évolution.

Si l'accrossement du nombre de bœufs engraissés est vivement souhaitable pour l'approvisionnement de Tananarive dont la population augmente très vite, dans le cadre des exploitations du type de celles d'Ampahimanga, Il faut craindre que cela ne soit possible que par une modification profonde des traditions, voire une modiflcation des structures des exploitations.

II n'en est peut-être pas de même partout ailleurs.

\section{SUMMARY}

The zebu cattle fattening in the area of Tananarive by the « ox of pit » method

\footnotetext{
Near Tananarive, lean zebu cattle are fattened being permed in «pits》 excavated in the hillsides round the villages. From december to march, the animals are fattened with herb, from march to july with growing again of rice, leaves of sweet potatoes and manioc. The animals become very fats with a carcase yjeld reaching 65 p. 100 . This activity associated with the rice culture
} 
should be intensified, but the small dimensions of the holdings seem to be $a$ lımiting facior in this area.

\section{RESUMEN}

\section{El engorde de los cebues en la región de Tananarive mediante la técnica del « buey de foso»}

En las cercanias de Tananarive, se engordan los cebues flacos instalandolos en « fosos » excavados en el costado de cuesta alrededor de los pueblos. De diciembre a marzo, se alimentan los animales con hierba, luego, de marzo a julı con rebrotas de arroz, hojas de patatas y mandioca. Los anımales se hacen muy gruesos con un rendimiento a la canal llegando a 65 p. 100 . Se necesitaria multiplicar dicha ganaderıa assaciada con el cultivo de arroz, pero la exiguidad de las propiedades parece ser un factor limitando en la región considerada. 